\title{
Intrinsic Orientation of Hydroxyapatite Grains on the Surface of Dense Pellets Produced by Uniaxial Pressing
}

\author{
Rafael Rabelo de Carvalho ${ }^{a, b}$, Leila Melo da Silva ${ }^{a}$, Euler Araujo dos Santos ${ }^{a, b} *$ [i] \\ aPrograma de Pós-Graduação em Ciência e Engenharia de Materiais - P2CEM, Universidade Federal \\ de Sergipe, Av. Marechal Rondon, s/n, CEP 49100-000, São Cristóvão, SE, Brasil \\ ${ }^{b}$ Departamento de Ciência e Engenharia de Materiais, Universidade Federal de Sergipe, Av. Marechal \\ Rondon, s/n, CEP 49100-000, São Cristóvão, SE, Brasil
}

Received: October 31, 2018; Revised: January 15, 2019; Accepted: March 09, 2019

\begin{abstract}
Bioceramics synthesized in laboratories must undergo biological tests that consider the final format and all the processing steps involved in the production of the final device. However, in many cases, the synthesized materials are characterized in formats very different from those that will ultimately be implanted into the body. Once living cells are directly cultured on surfaces, a mismatch between bulk and surface microstructural characterizations often leads to the misinterpretation of biological responses. Therefore, our objective in this work was to determine whether and at what level significant microstructural changes can occur between the surface and the bulk of hydroxyapatite pellets produced by uniaxial pressing followed by calcination. Our results showed that the as-synthesized hydroxyapatite crystals had a preferential orientation along the $\left[\begin{array}{lll}0 & 0 & 1\end{array}\right]$ direction. The calcination process followed by grain growth inhibited this texture feature. However, on the pellet surfaces, the initial orientation was preserved. At the same time, crystallite growth was inhibited, and the cell unit volume of the crystals on the surface was significantly lower than that of the crystals in the bulk. These results demonstrated that the crystallographic features on the surface of hydroxyapatite pellets can be completely different from those observed for the bulk.
\end{abstract}

Keywords: Hydroxyapatite, uniaxial pressing, texture, crystal structure, surface.

\section{Introduction}

When novel calcium phosphate bioceramics are synthesized via precipitation in aqueous media, the obtained powders are processed in different ways to produce final devices that can be used in applications such as biological assays ${ }^{1-4}$. In general, dense devices are obtained after the as-synthesized powder is subjected to pressing followed by calcination at a high temperature ${ }^{5}$. Calcination increases the mechanical strength of the devices, preventing their collapse when in contact with cell culture media or even when implanted in living tissue.

In biological assays, cells are cultured directly onto the surface of these calcined devices, and communication between the cells and the bioceramic material happens through the surface ${ }^{5-9}$. Therefore, thorough characterization of the surface crystalline structure is the most appropriate means to study the effect of the microstructure of the biomaterial on the cellular response ${ }^{9}$. However, in most cases, the crystallographic characterization of such bioceramics is performed on the as-synthesized powder after calcination $1,2,10,11$ and not on the dense device surface. Calcined devices have been taken into account in only a few cases; however, the $\mathrm{X}$-ray diffraction (XRD) analyses were still focused on bulk characterization and not on the surfaces of the devices themselves ${ }^{12-14}$. Therefore, several important studies on the biocompatibility of bioceramics have consistently assumed that the biological and crystallographic characteristics of the systems are unchanged between the bulk and the surface.

Uniaxial pressing processes can generate zones of different pressures that can induce notable density heterogeneity in the ceramic body ${ }^{15}$. Further, since the bulk material and the surface are exposed differently to the furnace atmosphere during calcination, the reactions that occur in the bulk can be quite different from those observed on the surface. All of these possible changes can affect cellular behavior since cells are sensitive to micro- and nanostructural heterogeneities on the surface. Therefore, important questions must be asked: is the crystalline structure of hydroxyapatite in dense devices the same in the bulk as on the surface? If not, what changes occur during the calcination process? The same questions can be asked for other ceramic systems where the surface is extremely important in determining the obtained properties ${ }^{16}$. To answer these questions, in this work, we propose a means of verifying the main crystallographic variations that can affect hydroxyapatite bioceramic materials submitted to uniaxial pressing and calcination by comparing the bulk and surface using XRD, glancing incidence X-ray diffraction (GIXRD), Rietveld refinement and transmission electron microscopy (TEM). 


\section{Materials and Methods}

A hydroxyapatite powder was obtained by the precipitation method ${ }^{17-19}$ in aqueous solution through a reaction between phosphoric acid $\left(0.100 \mathrm{~mol} \mathrm{~L}^{-1}\right)$ and calcium hydroxide $\left(0.167 \mathrm{~mol} \mathrm{~L}^{-1}\right)$. The reaction was conducted at $60^{\circ} \mathrm{C}$ under stirring, and the $\mathrm{pH}$ was maintained at 10 by the addition of potassium hydroxide $\left(0.1 \mathrm{~mol} \mathrm{~L}^{-1}\right)$. After $24 \mathrm{~h}$, the precipitate was filtered, washed with distilled water, dried at $110^{\circ} \mathrm{C}$ for $24 \mathrm{~h}$, and sieved $(106 \mu \mathrm{m})$ to obtain a powder. The reagents were purchased from Sigma-Aldrich and had purity values above $99 \%$.

The precipitated hydroxyapatite powder was processed under three distinct conditions:

a. The as-synthesized powder was calcined at $1150^{\circ} \mathrm{C}$ for $4 \mathrm{~h}$ at a heating rate of $2.8^{\circ} \mathrm{C} / \mathrm{min}$;

b. Dense pellets approximately $10 \mathrm{~mm}$ in diameter and $1.0 \mathrm{~mm}$ in height were made by uniaxial pressing of the synthesized powder under a pressure of 216 $\mathrm{MPa}$. The pellets were then calcined for $4 \mathrm{~h}$ at $1150^{\circ} \mathrm{C}$ at a heating rate of $2.8^{\circ} \mathrm{C} / \mathrm{min}$;

c. The pellets produced as described in item $b$ were ground and sieved $(106 \mu \mathrm{m})$ to obtain a new powder for further characterization.

The structural state of the hydroxyapatite powder before and after processing was studied by XRD in a RIGAKU MINIFLEX powder diffractometer $(\mathrm{CuK} \alpha \lambda=1.5405 \AA)$. The powders were scanned under Bragg-Brentano geometry from $20^{\circ}$ to $55^{\circ}$, with a step size of $0.05^{\circ}$ and a scanning speed of $1 \% \mathrm{~min}$. The surfaces of the dense pellets were analyzed by GIXRD in a Shimadzu XRD 6000 diffractometer $(\mathrm{CuK} \alpha \lambda=1.5405 \AA$ ) equipped with a low-angle apparatus. Diffraction was performed with a fixed incidence angle $(\theta)$ at $1.2^{\circ}$, a detection angle $(2 \theta)$ varying from $20^{\circ}$ to $55^{\circ}$ with a step size of $0.01^{\circ}$, and an acquisition time of $1 \mathrm{~s}$ per point.

The diffraction patterns were refined using the Rietveld method, which allowed us to follow the changes in the lattice parameters, texture (March/Dollase parameter) and crystallite size of the hydroxyapatite crystals before and after processing. The as-synthesized and calcined powders were also analyzed by TEM in JEOL 2100F and JEOL 2000FX electron microscopes operating at $200 \mathrm{kV}$. After calcination, the surface of the pellets was examined by scanning electron microscopy (SEM) using a JEOL JSM 6460LV microscope.

\section{Results and Discussion}

From the TEM images, the synthesized hydroxyapatite particles are needle shaped, with sizes varying between 50 and $200 \mathrm{~nm}$ (Fig. 1a). These particles are formed in some cases by single crystals and in other cases by the agglomeration of several crystals. This morphology is a typical characteristic of hydroxyapatite synthesized via aqueous precipitation. As- synthesized hydroxyapatites exhibit preferential orientation in the crystallographic direction [ $\left[\begin{array}{lll}0 & 0 & 1\end{array}\right]$, i.e., along the crystal $c$-axis ${ }^{20,21}$. Partial doping with $\mathrm{CO}_{3}^{2}, \mathrm{H}_{2} \mathrm{O}$, and several metallic ions intensifies the texturing of the as-synthesized hydroxyapatite crystals ${ }^{19,22}$.

Calcination at a high temperature allows particle coalescence by the known process of grain coarsening ${ }^{15,21,23}$. According to the Ostwald ripening mechanism ${ }^{15,20,24,25}$, smaller particles tend to transfer matter to larger ones, feeding grain growth. This process is well demonstrated by comparing the TEM images obtained from hydroxyapatite powders before (Fig. 1a) and after calcination (Fig. 1b). The elongated particles observed before calcination are replaced by spherical particles. This change indicates that calcination not only produces grain growth but also reduces the texture of the crystals.

To better evaluate these changes at a structural level, we analyzed the XRD patterns of powders before and after calcination. The powder before calcination exhibits a typical diffraction pattern of a hydroxyapatite phase (151414-ICSD). No other phase is observed beyond a poorly crystalline hydroxyapatite phase. Low crystallinity can be observed by the broadening of the diffraction peaks. After calcination, the peaks become sharper, and a new phase appears: $\mathrm{CaO}$ ( $\sim 0.5$ wt. \%) (52783-ICSD). This phase is associated with transformations occurring in hydroxyapatites with a $\mathrm{Ca} / \mathrm{P}$ ratio higher than $1.67^{22}$. The excess of $\mathrm{Ca}^{2+}$ ions tends to react with oxygen at high temperatures, which generates calcium oxide. As observed previously, as-synthesized hydroxyapatite generally contains $\mathrm{CO}_{3}{ }^{2-}$ that replaces $\mathrm{PO}_{4}{ }^{3-}$ groups (B-type doping), which increases the $\mathrm{Ca} / \mathrm{P}$ ratio.

The question thus far has been whether processing the original powder via uniaxial pressing can lead to significant structural changes to the original hydroxyapatite structure after calcination. To answer this question, the as-synthesized powder was used to produce pellets via uniaxial pressing followed by calcination under the same conditions as those previously used for the calcined powder. A portion of the pellets was ground for further analysis via XRD. The other portion was kept intact so that we could analyze the structural modifications on the surface after processing.

SEM images reveal the dense surface of the pellets after calcination (Fig. 2). Faceted grains with several sizes are observed on the surface. The diffraction pattern of the powder obtained from the calcined pellets after being ground is similar to that obtained for the as-synthesized powder after calcination (Fig. 1). Therefore, the pressing process does not significantly affect the crystalline structure of the hydroxyapatite. A similar amount of $\mathrm{CaO}(\sim 0.4 \mathrm{wt} . \%)$ was calculated from Rietveld refinement, confirming the small influence of the pressing process on the crystals. Moreover, the GIXRD diffraction pattern of the pellets demonstrates that textured grains formed on the surface. The peak related to the ( $\left.\begin{array}{lll}0 & 0 & 2\end{array}\right)$ plane of hydroxyapatite remarkably decreased, while 


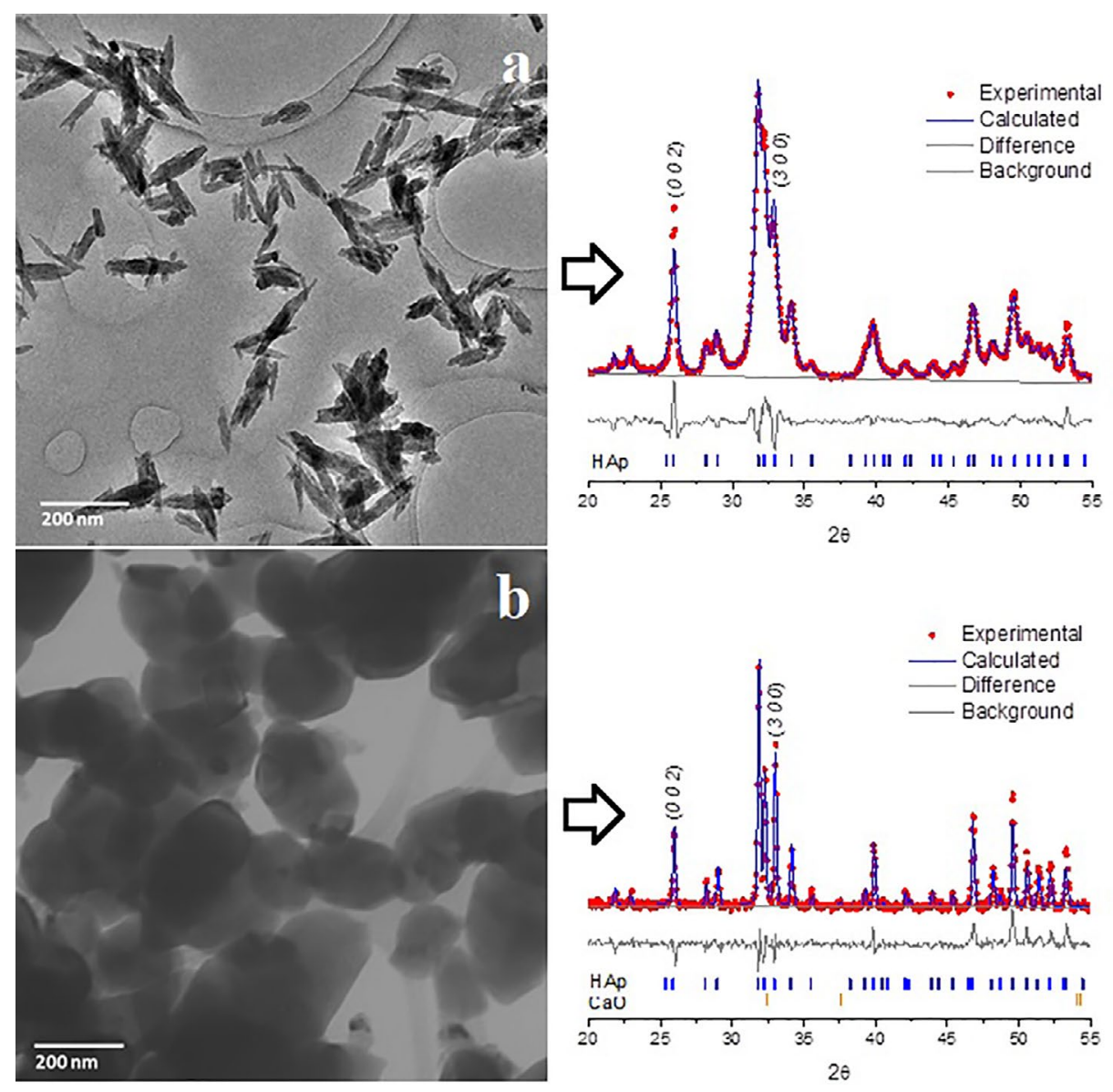

Figure 1. TEM images of (a) as-synthesized hydroxyapatite (HA) particles and (b) the same particles after calcination at $1150^{\circ} \mathrm{C}$ for $4 \mathrm{~h}$. Rietveld refinement of the XRD patterns obtained from the as-synthesized HA $(G o F: 3.05)$ and after calcination $(G o F: 2.46)$ are shown.

the peak related to the orthogonal $\left(\begin{array}{lll}3 & 0 & 0\end{array}\right)$ plane increased. This behavior indicates a preferential orientation of the surface grains in the $\left[\begin{array}{lll}0 & 0 & 1\end{array}\right]$ direction ${ }^{26}$.

The crystallite sizes were measured via the Scherrer equation ${ }^{27,28}$ along the $\left[\begin{array}{lll}0 & 0 & 1\end{array}\right]$ and $\left[\begin{array}{llll}1 & 0 & 0\end{array}\right]$ directions from the $\left(\begin{array}{lll}0 & 0 & 2\end{array}\right)$ and $\left(\begin{array}{lll}3 & 0 & 0\end{array}\right)$ planes, respectively. These planes were chosen because they do not present important overlaps and are orthogonal to each other. The average values are shown in Fig. 3. Crystallite growth is favored after calcination and inhibited on the surface compared to the bulk. The texture of the grains was evaluated by refining the March/Dollase parameter and assuming a typical orientation in the $\left[\begin{array}{lll}0 & 0 & 1\end{array}\right]$ direction. When this parameter is 1.0 , the orientation in the $[h$ $\mathrm{kl}$ ] direction is completely random. Values smaller than 1.0 mean a preferred orientation in the $[h \mathrm{kl}]$ direction. Values larger than 1.0 mean that orientation in the $[h k l]$ direction is avoided. The as-synthesized hydroxyapatite particles are oriented in the $\left[\begin{array}{lll}0 & 0 & 1\end{array}\right]$ direction. After calcination, the particles become more random as the parameter approaches 1.0. This behavior seems to be induced only by the calcination process, as the values for the pressed and nonpressed powders are similar. However, on the surface of the pressed pellets, the parameter is larger than 1.0 , indicating that orientation in the $\left[\begin{array}{lll}0 & 0 & 1\end{array}\right]$ direction is avoided.

If we consider the use of a fixed glancing incidence angle almost parallel to the surface, the increase in this parameter indicates that the $\left(\begin{array}{lll}0 & 0\end{array}\right)$ planes on the surface are preferentially perpendicular to the X-ray beam, which reduces their signal at the detector ${ }^{26}$. Conversely, the scattering from the $(30$ 0 ) planes parallel to the X-ray beam is stronger, which is in fact observed in the GIXRD pattern (Fig. 2). Therefore, the increase in the March/Dollase parameter means that the grains are oriented along the $\left[\begin{array}{lll}0 & 0 & 1\end{array}\right]$ direction on the pellet surface. The initial orientation observed for the as-synthesized hydroxyapatite is preserved on the pellet surfaces. One can conclude that both crystallite size and orientation are strongly affected by the region where crystallites are found in the pellets. This relationship is further demonstrated by the calculated lattice parameters. Calcination tends to decrease the cell unit volume compared with that of the as-synthesized hydroxyapatite. This reduction is even more pronounced for crystals on the pellet surfaces, confirming that the grains 


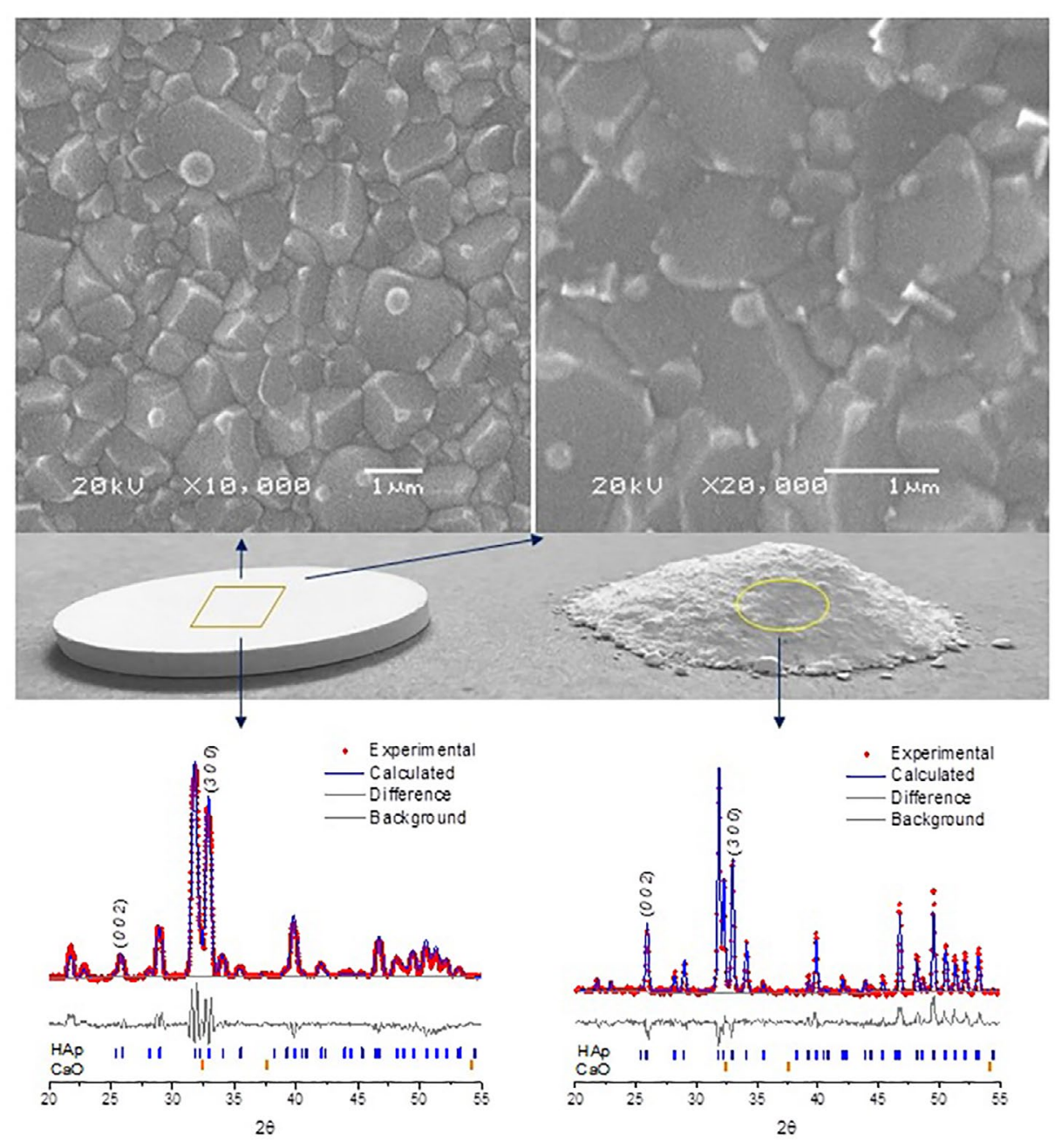

Figure 2. Macro images of pellets obtained after pressing/calcination and in powder form after grinding. Details on the pellet surfaces can be observed in scanning electron microscopy (SEM) images at different magnifications. The crystallographic parameters of both the surface and bulk materials can be compared in X-ray diffraction (XRD) patterns. Rietveld parameters: GoF 1.45 (left), GoF 2.16 (right).

on the surface and in the bulk have remarkably different crystallographic characteristics.

All these behaviors seem to be entirely dependent on the original condition of the particles used in the uniaxial pressing process. Since the as-synthesized particles have an intrinsic elongation (Fig. 1a), their packing in the bulk and on the surface is different. In the bulk, the elongated particles are randomly distributed when they are poured into the steel die, which results in structural isotropy (Fig. 4). On the top and bottom surfaces, the elongated particles tend to lie flat due to the gravitational force. After pressing, the particles on the top surface become oriented in parallel planes. Despite the random orientation of elongated particles in each plane, the arrangement of particles in parallel makes all crystal planes in the $\left[\begin{array}{lll}0 & 0 & 1\end{array}\right]$ direction almost perpendicular to the incident X-ray beam in GIXRD analysis, as discussed above.

The main implication of these results is that cells can respond differently to surfaces that exhibit topographic or crystallographic anisotropy. Several studies have demonstrated that oriented surface grooves at the nano- and microscale can induce polarized growth or a preferential cell spreading direction ${ }^{29-33}$. This phenomenon is known as contact guidance and occurs through an important cytoskeletal reorganization that implies changes in the adhesion, proliferation and differentiation of the cells ${ }^{34}$. It has also been demonstrated that cells can exhibit different behavior when cultured on different faces of the same crystal ${ }^{35,36}$. If each crystal face exhibits unique chemical terminations and atomic packing, the surface energies attributed to the faces can diverge. Surface energy directly influences two important phenomena in cell/biomaterial interactions ${ }^{4,37,38}$ : protein adsorption and cell attachment. Therefore, careful and precise surface characterization is essential for correctly describing cell behavior on biomaterial devices. 

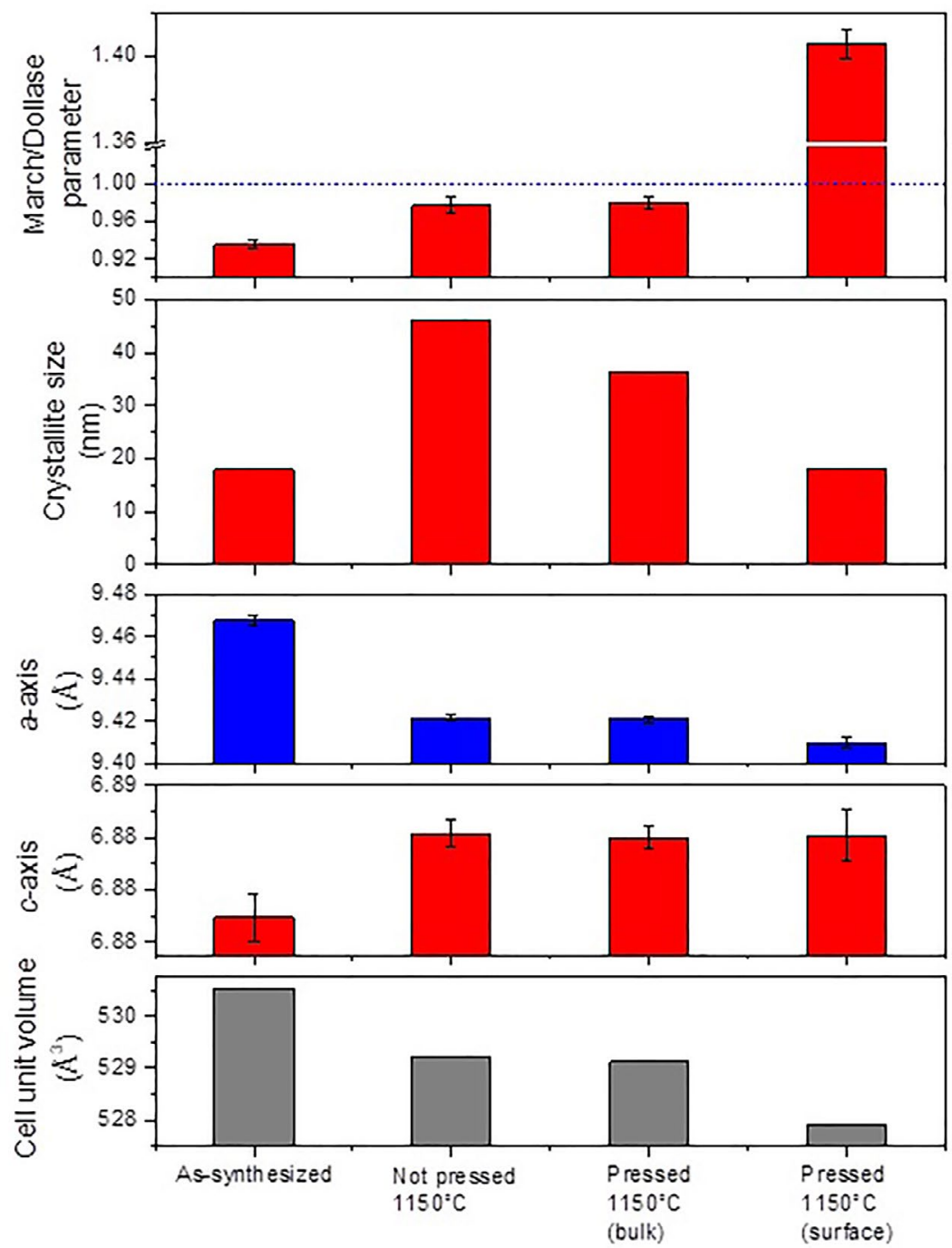

Figure 3. Lattice parameters, cell unit volume, crystallite size and orientation of the hydroxyapatite crystals throughout processing, as measured from Rietveld refinement data.
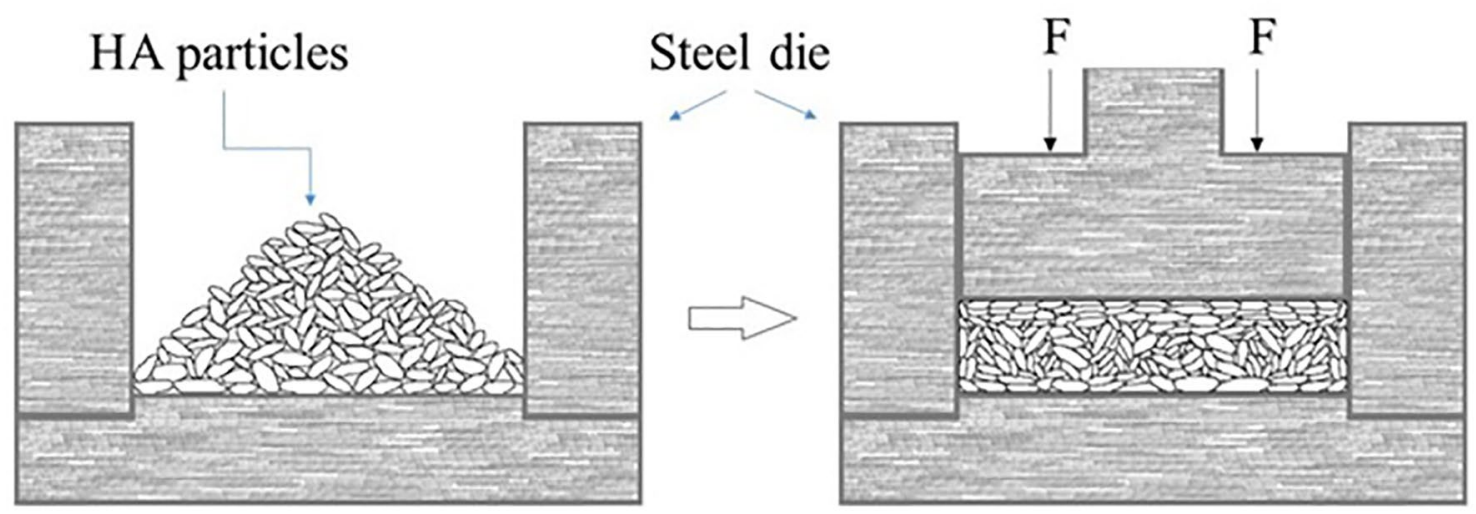

Figure 4. Schematic illustration of the HA particle arrangement after being poured into the steel die (left) and after being pressed (right) into pellets. 


\section{Conclusions}

As-synthesized hydroxyapatite crystals had a preferential orientation along the $\left[\begin{array}{lll}0 & 0 & 1\end{array}\right]$ direction. The calcination process followed by grain growth inhibited this texture feature. However, on the pellet surface, the initial orientation of the crystal was preserved. The crystallite growth and cell unit volume were significantly reduced for the crystals on the surface relative to those in the bulk. These results demonstrate that the crystallographic features on the surface of hydroxyapatite pellets can be completely different from those observed for the bulk, which may compromise the interpretation of cellular behavior generally attributed to the bulk material.

\section{Acknowledgments}

The authors acknowledge financial support from the Brazilian research agencies FAPITEC/SE, CAPES-Cofecub and CNPq, as well as IPCMS/CNRS-Strasbourg and COPPE/ UFRJ-Rio de Janeiro for the use of microscopy facilities.

\section{References}

1. Bang LT, Ramesh S, Purbolaksono J, Ching YC, Long BD, $\mathrm{Chandran} \mathrm{H}$, et al. Effects of silicate and carbonate substitution on the properties of hydroxyapatite prepared by aqueous coprecipitation method. Materials \& Design. 2015;87:788-796.

2. Alshemary AZ, Akram M, Goh YF, Tariq U, Butt FK, Abdolahi A, et al. Synthesis, characterization, in vitro bioactivity and antimicrobial activity of magnesium and nickel doped silicate hydroxyapatite. Ceramics International. 2015;41(9 Pt B):1188611898.

3. dos Santos EA, Farina M, Soares GA, Anselme K. Chemical and topographical influence of hydroxyapatite and $\beta$-tricalcium phosphate surfaces on human osteoblastic cell behavior. Journal of Biomedical Materials Research - Part A . 2009;89A(2):510520.

4. dos Santos EA, Farina M, Soares GA, Anselme K. Surface energy of hydroxyapatite and beta-tricalcium phosphate ceramics driving serum protein adsorption and osteoblast adhesion. Journal of Materials Science. Materials in Medicine. 2008;19(6):2307-2316.

5. Canillas M, Pena P, de Aza AH, Rodríguez MA. Calcium phosphates for biomedical applications. Boletín de la Sociedad Española de Cerámica y Vidrio. 2017;56(3):91-112.

6. Anselme K. Osteoblast adhesion on biomaterials. Biomaterials. 2000;21(7):667-681.

7. dos Santos EA, Moldovan S, Mateescu M, Faerber J, Acosta $\mathrm{M}$, Pelletier H, et al. Physical-chemical and biological behavior of an amorphous calcium phosphate thin film produced by RFmagnetron sputtering. Materials Science and Engineering: $C$. 2012;32(7):2086-2095.
8. Anselme K, Bigerelle M. Modelling approach in cell/material interactions studies. Biomaterials. 2006;27(8):1187-1199.

9. Anselme K, Ponche A, Bigerelle M. Relative influence of surface topography and surface chemistry on cell response to bone implant materials. Part 2: Biological aspects. Proceedings of the Institution of Mechanical Engineers, Part H: Journal of Engineering in Medicine. 2010;224(12):1487-1507.

10. Gomes S, Vichery C, Descamps S, Martinez H, Kaur A, Jacobs A, et al. Cu-doping of calcium phosphate bioceramics: From mechanism to the control of cytotoxicity. Acta Biomaterialia. 2018;65:462-474.

11. Marques CF, Olhero S, Abrantes JCC, Marote A, Ferreira S, Vieira SI, et al. Biocompatibility and antimicrobial activity of biphasic calcium phosphate powders doped with metal ions for regenerative medicine. Ceramics International. 2017;43(17):15719-15728.

12. Gokcekaya O, Ueda K, Narushima T, Ergun C. Synthesis and characterization of Ag-containing calcium phosphates with various $\mathrm{Ca} / \mathrm{P}$ ratios. Materials Science and Engineering: $C$. 2015;53:111-119.

13. Xue W, Dahlquist K, Banerjee A, Bandyopadhyay A, Bose S. Synthesis and characterization of tricalcium phosphate with $\mathrm{Zn}$ and Mg based dopants. Journal of Materials Science: Materials in Medicine. 2008;19(7):2669-2677.

14. Begam H, Kundu B, Chanda A, Nandi SK. MG63 osteoblast cell response on $\mathrm{Zn}$ doped hydroxyapatite (HAp) with various surface features. Ceramics International. 2017;43(4):3752-3760.

15. Chiang YM, Birnie D, Kingery WD. Physical Ceramics: Principles for Ceramic Science and Engineering. New York: John Wiley \& Sons; 1997.

16. Zhang J, Li M, Feng Z, Chen J, Li C. UV Raman Spectroscopic Study on $\mathrm{TiO}_{2}$. I. Phase Transformation at the Surface and in the Bulk. The Journal of Physical Chemistry B. 2006;110(2):927935.

17. Fihri A, Len C, Varma RS, Solhy A. Hydroxyapatite: A review of syntheses, structure and applications in heterogeneous catalysis. Coordination Chemistry Reviews. 2017;347:48-76.

18. da Silva LM, Menezes DS, Almeida LE, Anselme K, Dentzer J, dos Santos EA. The role of the counter-ions present in syntheses on the thermal stabilization of strontium and/or calcium apatites. Materials Science and Engineering: B. 2015;199:77-86.

19. Simões FB, da Silva LM, Fonseca TO, Almeida LE, dos Santos EA. Isolated effects of microwave radiation on the solid-liquid interface reactions between hydroxyapatite nanocrystals and silver ions. Ceramics International. 2018;44(14):16960-16971.

20. Dhand V, Rhee KY, Park SJ. The facile and low temperature synthesis of nanophase hydroxyapatite crystals using wet chemistry. Materials Science and Engineering: C. 2014;36:152159.

21. Gandhi AA, Gunning RD, Ryan KM, Tofail SAM. The Role of Texturing and Densification on Optical Transmittance of Hydroxyapatite Ceramics. Journal of the American Ceramic Society. 2010;93(11):3773-3777. 
22. Elliott JC. Structure and Chemistry of the Apatites and Other Calcium Orthophosphates. Amsterdam: Elsevier; 1994.

23. Wu J, Ruan C, Ma Y, Wang Y, Luo Y. Vital role of hydroxyapatite particle shape in regulating the porosity and mechanical properties of the sintered scaffolds. Journal of Materials Science \& Technology. 2018;34(3):503-507.

24. Mary IR, Sonia S, Viji S, Mangalaraj D, Viswanathan C, Ponpandian N. Novel multiform morphologies of hydroxyapatite: Synthesis and growth mechanism. Applied Surface Science. 2016;361:25-32.

25. Ren F, Ding Y, Ge X, Lu X, Wang K, Leng Y. Growth of one-dimensional single-crystalline hydroxyapatite nanorods. Journal of Crystal Growth. 2012;349(1):75-82.

26. Zhu X, Sakka Y. Textured silicon nitride: processing and anisotropic properties. Science and Technology of Advanced Materials. 2008;9(3):033001.

27. Ince T, Kaygili O, Tatar C, Bulut N, Koytepe S, Ates T. The effects of Ni-addition on the crystal structure, thermal properties and morphology of Mg-based hydroxyapatites synthesized by a wet chemical method. Ceramics International. 2018;44(12):1403614043.

28. Moreira MP, de Almeida Soares GD, Dentzer J, Anselme K, de Sena LÁ, Kuznetsov A, et al. Synthesis of magnesium- and manganese-doped hydroxyapatite structures assisted by the simultaneous incorporation of strontium. Materials Science and Engineering: C. 2016;61:736-743.

29. Dalby MJ, Riehle MO, Yarwood SJ, Wilkinson CDW, Curtis ASG. Nucleus alignment and cell signaling in fibroblasts: response to a micro-grooved topography. Experimental Cell Research. 2003;284(2):272-280.
30. Deligianni DD, Katsala ND, Koutsoukos PG, Missirlis YF. Effect of surface roughness of hydroxyapatite on human bone marrow cell adhesion, proliferation, differentiation and detachment strength. Biomaterials. 2000;22(1):87-96.

31. Eisenbarth E, Linez P, Biehl V, Velten D, Breme J, Hildebrand HF. Cell orientation and cytoskeleton organisation on ground titanium surfaces. Biomolecular Engineering. 2002;19(2-6):233-237.

32. Raimbault $\mathrm{O}$, Benayoun $\mathrm{S}$, Anselme $\mathrm{K}$, Mauclair $\mathrm{C}$, Bourgade $\mathrm{T}$, Kietzig AM, et al. The effects of femtosecond laser-textured Ti6Al-4V on wettability and cell response. Materials Science and Engineering: C. 2016;69:311-320.

33. dos Santos EA, Linhares ABR, Rossi AM, Farina M, Soares GDA. Orientation of Human Osteoblast Cells on Biphasic Calcium Phosphates Tablets with Undulated Topography. Key Engineering Materials. 2004;254-256:793-796.

34. Dalby MJ. Topographically induced direct cell mechanotransduction. Medical Engineering \& Physics. 2005;27(9):730-742.

35. Zimmerman E, Addadi L, Geiger B. Effects of Surface-Bound Water and Surface Stereochemistry on Cell Adhesion to Crystal Surfaces. Journal of Structural Biology. 1999;125(1):25-38.

36. Hanein D, Geiger B, Addadi L. Fibronectin adsorption to surfaces of hydrated crystals. An analysis of the importance of bound water in protein-substrate interactions. Langmuir. 1993;9(4):1058-1065.

37. Kilpadi KL, Chang PL, Bellis SL. Hydroxylapatite binds more serum proteins, purified integrins, and osteoblast precursor cells than titanium or steel. Journal of Biomedical Materials Research. 2001;57(2):258-267.

38. Kennedy S, Washburn N, Simonjr C, Amis E. Combinatorial screen of the effect of surface energy on fibronectin-mediated osteoblast adhesion, spreading and proliferation. Biomaterials. 2006;27(20):3817-3824. 